\title{
FLUID-STRUCTURE INTERACTION ANALYSIS APPLIED TO THERMAL BARRIER COATED COOLED ROCKET THRUST CHAMBERS WITH SUBSEQUENT LOCAL INVESTIGATION OF DELAMINATION PHENOMENA
}

\author{
D. S. C. Kowollik, P. Horst, and M. C. Haupt \\ Institute of Aircraft Design and Lightweight Structures \\ TU Braunschweig \\ 35 Herrmann-Blenk-Str., Braunschweig 38106, Germany
}

\begin{abstract}
The aim of this work is to investigate numerically thermal barrier coating (TBC) systems applied to realistic rocket thrust chamber conditions. A global full parametric three-dimensional (3D) modeling approach for cooled rocket thrust chambers is presented to be able to simulate the fluid-structure interaction (FSI) phenomena involved. In a subsequent analysis step, realistic mechanical and thermal boundary conditions are extracted from critical design regions of the global model and applied to a local finite element model (FEM) to analyze possible TBC delaminations by means of a Fracture Mechanics (FM) approach.
\end{abstract}

\section{INTRODUCTION}

The wall of modern liquid rocket engines is exposed to extreme thermal loadings. In current designs, high aspect ratio cooling channels are milled in the copper liner, where the chamber wall is approximately $1 \mathrm{~mm}$ thick. This setup is kept intact only for a few engine cycles. A protection of the thin copper cooling structure is a promising strategy to enhance thrust chamber life. Thermal barrier coatings are widely used in applications at elevated temperatures, e. g., automotive, gas turbines, and aeroengines. The transfer of TBC systems to rocket applications offers great potential to reduce coolant temperature and pressure loss in the cooling cycle based on the reduction of heat flux acting on the hot gas surface inside the combustion chamber. A careful design is necessary to cope with the transient boundary conditions, under which TBCs tend to spall, delaminate, and crack.

This is an Open Access article distributed under the terms of the Creative Commons Attribution License 2.0, which permits unrestricted use, distribution, and reproduction in any medium, provided the original work is properly cited. 
The response of the cooling channel can only be addressed through an adequate prediction of the in-service conditions. Chemical reactions during the combustion process, fluid flow in the thrust chamber and cooling channels, heat transfer between the involved domains and the thermomechanical coupling in the structure make up a complex system. The thermal and pressure loads generated by the hot gas and the cooling channel flow are essential for the prediction of the cooling channel response.

Computer resources in the past were limited to the application of simplified models for coupled simulations. In [1], the thermal interaction between a twodimensional (2D) structural model and a straight 3D cooling channel segment is analyzed. The interaction with the hot gas side is neglected, whereas experimental data of [2] serve as boundary condition. This simplified model is extended by a thermomechanical coupling strategy in [3]. A 3D coupled heat transfer analysis is presented by Liu et al. in [4], where the hot gas and cooling channel structure are modeled by a finite volume scheme. The cooling channel flow is included by a one-dimensional (1D) approach. Current simulation strategies were presented by Knab et al. in [5], where a coupled heat transfer simulation was performed between the hot gas side and the coolant flow. The hot gas side is modeled by an axisymmetric multiphase Navier-Stokes solver, whereas the cooling channel flow and the structure are analyzed by a $3 \mathrm{D}$ conjugate heat transfer model.

In the discipline of turbomachinery, a lot of studies focus on conjugate heat transfer analyses (see, e.g., $[6,7]$ ) which differ algorithmically in the details of the coupling strategy from the present approach. For example, in the work of Heidmann et al. [6], the interface conditions are transferred in a reverse manner compared to the approach that is followed in the present study, where heat flux is tranferred from the fluid analysis to the structural analysis and temperature is transfered from the structural side to the fluid side, respectively.

Concerning the present FSI analyses of the heat transfer problem in rocket thrust chambers, a similar approach is followed, whereas the present partitioned approach follows a more general scheme in developing a simulation environment which is flexible and capable to introduce different analysis codes for the individual disciplines involved. For example. in this study, the FSI analyses take the thermal interactions into account and the mechanical problem is analyzed subsequently including the converged thermal and mechanical loads. The present approach focuses on the integration of both involved physical couplings, the thermal and mechanical ones, in a unified FSI approach with a subsequent investigation of local fracture mechanisms.

This paper extends the previous studies by accounting for the full thermal and mechanical interactions between the hot flow field and the structure by applying TBC systems to a parametric 3D modeling approach, which is presented. A subsequent local investigation with applied boundary conditions of the global analyses gives first insight in the loading conditions acting on macroscopic delaminations being located at the critical throat region of the thrust chamber. 


\section{COUPLED SIMULATION APPROACH}

Analyzing cooled rocket thrust chambers demands for the consideration of thermal and mechanical fluid-structure interaction. The developed simulation environment ifls covers both physical couplings in a partitioned approach using individual codes for the involved subdomains fluid and structure. A detailed description of ifls can be taken from [8]. Validation studies of coupled problems analyzed by different individual code combinations are presented in [8-10].

The two coupling aspects that need to be considered applying a partioned approach for a steady-state analysis are the data transfer of the variables to be coupled through nonconforming surface discretizations and the solution of the equilibrium equations.

\subsection{Nonconforming Spacial Discretization at the Interface}

Data transfer between nonconforming surface discretizations at the coupling interface $\Gamma_{c}$ can be achieved in a conservative manner by applying a weak formulation to the continuity conditions [11], where the variations of the involved state variables $\phi$ (temperatures $T$ and deflections $u$ ) are weighted by Lagrange multipliers $\lambda_{\phi}$ as follows:

$$
\int_{\Gamma_{c}} \lambda_{T}\left(T^{(s)}-T^{(f)}\right) \mathrm{d} \Gamma ; \int_{\Gamma_{c}} \lambda_{u}\left(u^{(s)}-u^{(f)}\right) \mathrm{d} \Gamma .
$$

The indices $(s)$ and $(f)$ represent the fluid and the structural domains. In the discrete form, expressions (1) result in the matrix system

$$
\left.\phi^{(f)}\right|_{\Gamma}=\left.\mathbf{H} \phi^{(s)}\right|_{\Gamma}
$$

by using shape functions for the Lagrange multipliers and for the individual domain discretizations fluid and structure at the interface. The transformation matrix $\mathbf{H}$ can be used in its tranposed form in order to guarantee conservation of energy, if the consistent mechanical and thermal discrete fluxes are transferred normal to the interface:

$$
\left.\phi_{, \mathbf{n}}^{(f)}\right|_{\Gamma}=\left.\mathbf{H}^{\mathrm{T}} \phi_{, \mathbf{n}}^{(s)}\right|_{\Gamma} .
$$

If the mesh discretization does not differ significantly between the individual domains, the Dirac delta functions applied at the fluid interface nodes are the standard choice for the shape functions of the Lagrange multiplier.

Strong discretization mismatch will result in unphysical load peaks, which can be alleviated by a locally more accurate approach, where the fluid shape functions are used for the Lagrange multiplier leading to an approach comparable to the Mortar-technique (see [12]). For this transfer approach, an inversion of the sparse 
and positive definite matrix is necessary. In a generic transfer scheme, this matrix can be decomposed by the Cholesky factorization and, therefore, reused during each data transfer step of the FSI analysis. In systems with a large number of degrees of freedom, this decomposition is computational expensive. DualLagrange multipliers can be constructed depending on the fluid shape functions, which lead to a diagonalized matrix making the inversion process trivial [12].

\subsection{Solution of the Partitioned Formulation}

The solution of the coupled problem is obtained by the Dirichlet-Neumann iteration. Formulating the Dirichlet problem in terms of a Schur complement, one defines symbolically the fluid operator $\mathcal{F}$ as follows:

$$
\left.\mathcal{F} \phi^{(f)}\right|_{\Gamma}=\left.\phi_{, \mathbf{n}}^{(f)}\right|_{\Gamma}
$$

where $\phi$ represents the state variables and $\phi_{, \mathbf{n}}$ the flux at the coupling interface $\Gamma$. The inverse Schur complement is formulated for the structural problem; consequently, the Neumann problem is solved:

$$
\left.\phi_{, \mathbf{n}}^{(s)}\right|_{\Gamma}=\left.\left.\mathcal{S}^{-1} \phi^{(s)}\right|_{\Gamma} \longleftrightarrow \mathcal{S} \phi^{(s)}\right|_{\Gamma}=\left.\phi_{, \mathbf{n}}^{(s)}\right|_{\Gamma}
$$

For the classical Dirichlet-Neumann iteration, a relaxation scheme is applied to the state variables in each steady-state iteration step as follows:

$$
\phi_{k+1}=(1-\omega) \phi_{k}+\omega \mathcal{S}^{-1} \mathcal{F} \phi_{k},
$$

with the relaxation parameter $\omega$. For the coupled problem in the present work, a choice of a fixed $\omega=0.8$ was a proper choice to achieve a noticeable reduction of the residual during the fixed point iteration.

\section{PARAMETRIZED THRUST CHAMBER MODELING APPROACH}

Three-dimensional FSI analyses of cooled rocket thrust chambers are computationally expensive. Computational reduction can be achieved if symmetry conditions are used; consequently, the assumption of the present parametric approach is to model half of a cooling channel segment for the combustion chamber cooling circuit. The hot gas is modeled with periodic boundary conditions, which is shown in Fig. 1 where the coupling surfaces are defined. The data transfer between the hot gas and structural domain is achieved through an additional coupling surface $\Gamma_{s, h g}$ where the state and flux variables are mirrored to satisfy 


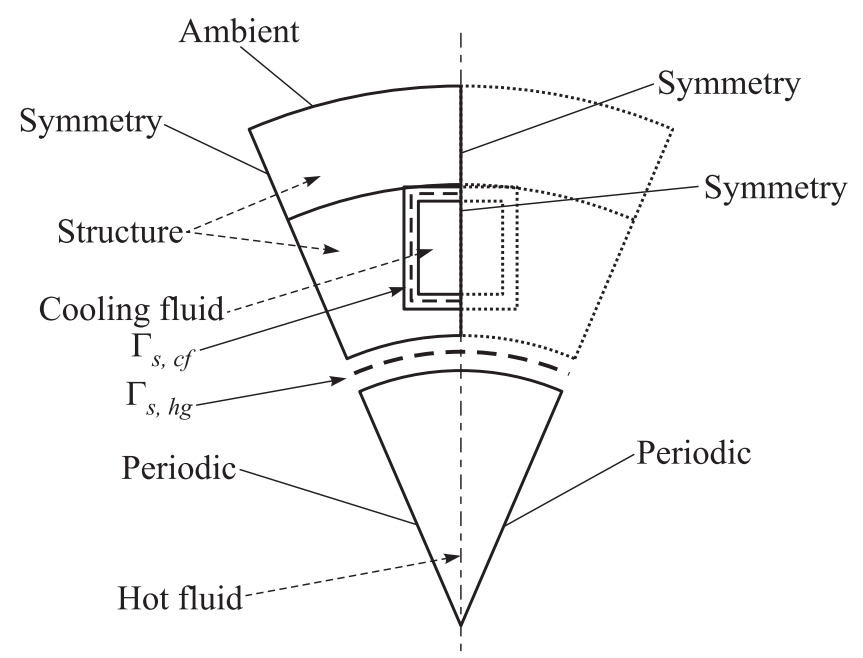

Figure 1 Definition of coupling surfaces

integrity of the applied boundary conditions in each iteration step. Accounting for all symmetry conditions in the different computational domains allows the simulation of the $3 \mathrm{D}$ state by the assumption of periodic repetition.

A software concept for parametrized modeling of thrust chamber designs and cooling channel setups was developed and is shown schematically in Fig. 2. The parametrization is reached from the thrust chamber contour and cooling channel design to the CAD modeling and, finally, to the grid generation of the hot gas, structure, and cooling channel flow field. The software architecture uses the Python interface to the preprocessor Abaqus/CAE. Python scripting allows an object oriented modular and reusable framework for the different components of the parametrized models. Additional software packages like Triangle [13] and TetGen [14] are used to discretize the unstructured part of the hybrid hot gas grid.

The class definition of ThrustChamberContour describes the complete rocket engine contour of Fig. $3 a$, where the nozzle is designed with the thrust optimized parabola (TOP) from Rao [15]. The TOP nozzle can be described by five independent variables: $r_{t d}, \theta_{n}, L, r_{e}$, and $\theta_{e}$. The angles $\theta_{n}$ and $\theta_{e}$ are evaluated through a bivariate spline whose input data were taken from Rao [15]. Even modern nozzles like the Vulcain and SSME (Space Shuttle Main Engine) nozzle can be studied by means of a parabolic contour [16].

The derived class ParamCooling Geom accounts for the chosen cooling circuit cross section definition shown in Fig. $3 b$. For the present cooling channel setup, the classes are derived for the different computational domains, which implement 


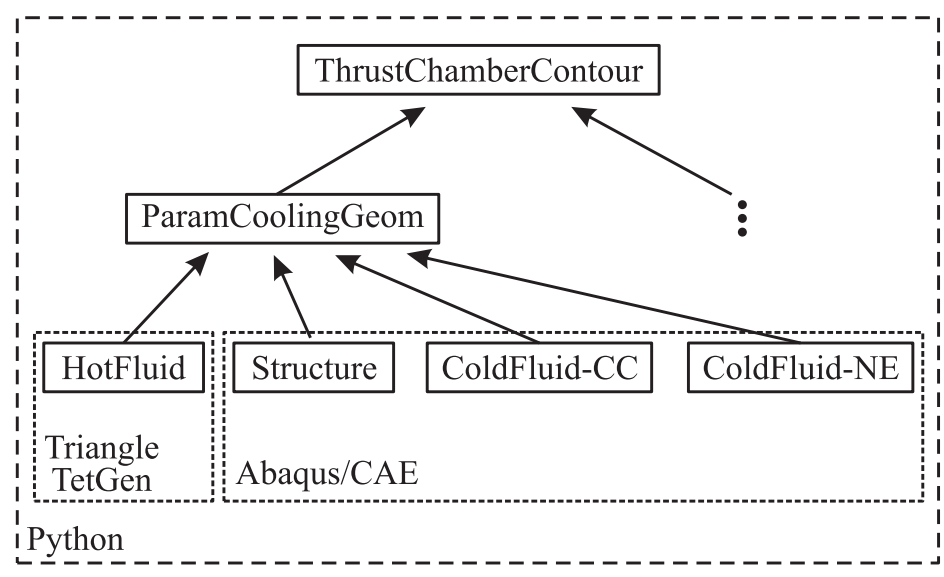

Figure 2 Software concept

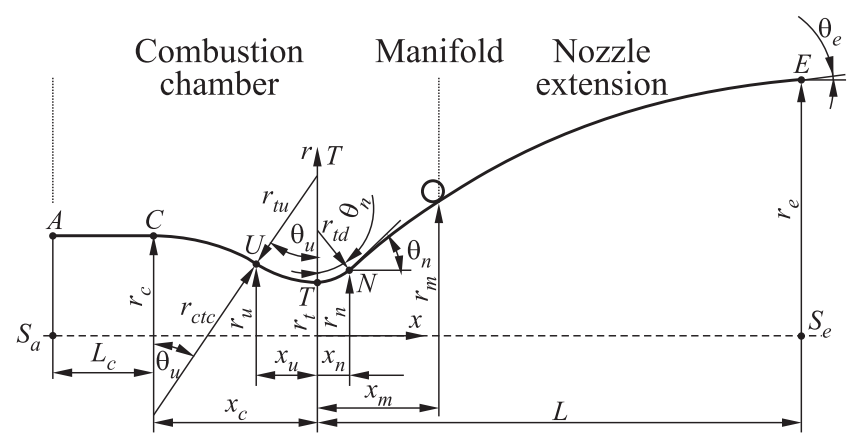

(a)

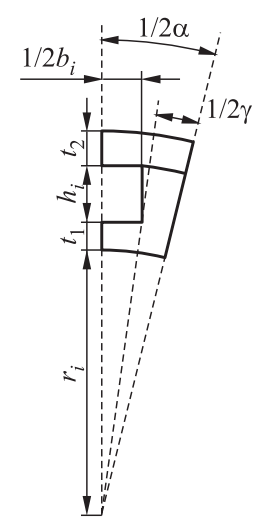

(b)

Figure 3 Parametrized model: $(a)$ thrust chamber contour; and (b) cooling channel cross section

the parametrized CAD and grid models. In this study, the analyzed subscale thrust chamber consists of three domains, namely, structure, hot gas, and the two cooling curcuits, one in the combustion chamber region and one in the nozzle extension. For both cooling circuits, a continously varying cooling channel geometry can be defined for the cross section sketched in Fig. $3 b$.

The structural model is able to account for TBC systems consisting of up to two individual materials, which can be seen in Fig. 4. The copper and nickel 


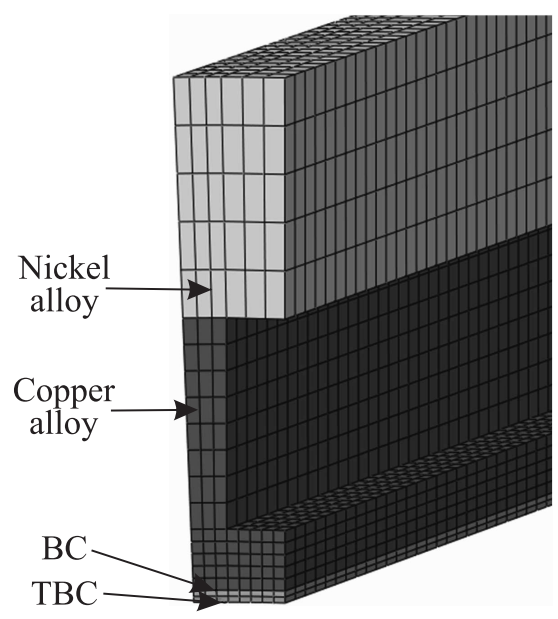

Figure 4 Close view of the material setup of the parametrized global structural model

material is modeled via 8 node linear solid elements and the thin TBC system materials are accounted for via 8 node linear solid shell elements.

\section{FLUID-STRUCTURE INTERACTION ANALYSES OF THERMAL BARRIER COATED COOLED ROCKET THRUST CHAMBERS}

Extracted general parameters of a 40-kilonewton $\mathrm{LOx} / \mathrm{H}_{2}$ subscale rocket thrust chamber defined by Astrium Space Transportation GmbH, Propulsion \& Equipment serve as input for the parametrized modeling approach. The thrust chamber features 80 cooling channels in the combustion chamber and 160 cooling channels in the nozzle extension.

On the hot gas side, a 3D steady Reynolds averaged Navier-Stokes (RANS) analysis using ideal gas law assuming finalized combustion is performed in each Dirichlet step of the static FSI analysis. For the fluid simulation, the DLR TAUCode [17] is used, which is an unstructured RANS solver based on the finite volume method. Reservoir pressure inflow conditions were computed with the preliminary design tool RPA (Rocket Propulsion Analysis) [18] and served as inlet conditions for the DLR TAU-Code. The temperature niveau computed with RPA reaches $3,502 \mathrm{~K}$ at a pressure level of $9.35 \mathrm{MPa}$. At the nozzle exit, supersonic outlet conditions are applied, while an isothermal wall is defined at the coupling surface. 
The turbulent effects are modeled by the original version of the SpalartAllmaras model implemented in the TAU-Code. The work of Tahsini [19] and Wang et al. [20] show a good agreement between the Spalart-Allmaras model, experiments, and higher class methods, e.g., Large Eddy Simulation (LES), concerning the applicabability of the one-equation turbulence model in the presence of streamwise curvature with the focus on heat transfer analysis, which is an important aspect for the hot gas analyses. Wang et al. analyzed flow over a sphere comparing LES, the Spalart-Allmaras model, data given by Meissner and Buttner, and two empirical Nusselt correlations, one recommended by McAdams [21] and the other recommended by Whitaker [22] for a Reynolds number range from $10^{3}$ to $1.5 \cdot 10^{5}$. Tahsini compared the Spalart-Allmaras model with experimental data for a wall jet tangentially entering the flow of a cylindrical channel. Tahsini determined the heat transfer coefficients downstream of the jet and demonstrated a good agreement compared to the experimental results published by Lebedev et al. [23]. Therefore, the Spalart-Allmaras model shows a good agreement between accuracy and computational efficiency for the present FSI analyses.

The hybrid grid of the present study consists of 590,833 grid points, 686,535 tetrahedra, and 785,686 prisms. The dimensionless $y+$ is adapted to a maximum of 0.57 at the thrust chamber throat. Figure 5 shows the typical Mach number profile of a TOP nozzle, where compression waves are generated at the intersection of the downstream arc and the parabola contour. These compression waves coalesce in an internal shock. Comparisons to Östlund approve these flow phenomena [16].

The steady-state heat transfer problem of the cooling channel structure is analyzed with the ABAQUS FE software. The resultant heatflux of the hot gas simulation is transferred as boundary condition to the coupling surface. Constant heat transfer coefficients are assumed for the two coolant circuits, whereas the heat transfer coefficient of $h_{f, \mathrm{CC}}=150 \mathrm{~kW} /\left(\mathrm{m}^{2} \mathrm{~K}\right)$ is applied for the combustion chamber circuit and $h_{f, \mathrm{NE}}=30 \mathrm{~kW} /\left(\mathrm{m}^{2} \mathrm{~K}\right)$ is applied for the nozzle extension circuit. For both circuits, the sink temperature is defined as $T=40 \mathrm{~K}$. Radi-

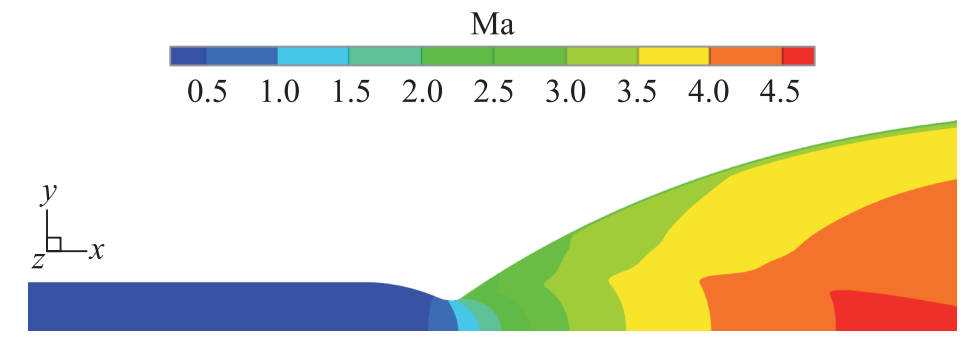

Figure 5 Mach number distribution at steady state of the coupled FSI analysis. 
ation effects in the combustion chamber are not considered so far. Radiation to ambient is computed for the outside facing surface of the cooling channel structure.

The resultant temperature distribution together with the mechanical loads are used as boundary conditions for the static stress/displacement analysis. At the inlet, the deflections in the axial direction are suppressed. Symmetric deformation is guaranteed by applying zero deflection normal to the symmetry planes sketched in Fig. 1. The material parameters of copper (substrate: $\mathrm{CuCr} 1 \mathrm{Zr}$ ), BC (bond coat: NiCrAlY) and TBC (top coat: zirconia) are taken from [24-26]. INCONEL alloy 600 [27] is used for the nickel jacket of the combustion chamber and the nozzle extension.

\subsection{Numerical Accuracy}

The accuracy of the above presented solution of the partioned FSI formulation is monitored during the Dirichlet-Neumann iterations. The iterative convergence is assessed to be sufficient once the L2 norm residual of the temperature interface conditions is reduced by at least four orders of magnitude. The interface conditions are transferred during each iteration once convergence of the individual solver codes was achieved likewise.

Grid convergence is analyzed for each parametrized domain grid, hot gas and structure separately. For the present study, the grid convergence index (GCI) is applied, which is a recommended method by ASME for evaluating the numerical uncertainty in fluid engineering [28]. The method is based on the Richardson extrapolation [29]. For a uniform assessment in this study, the GCI is also applied to the structural domain. Three different grid sizes are generated for each domain. The grid parameters of the individual meshes are listed in Table 1.

The cell edge distribution was approximately doubled in each refinement step. The point of interest of the present study is located at the nozzle throat; therefore, the field variables temperature and heat flux are investigated for the GCI evaluation. A constant temperature of $T=1600 \mathrm{~K}$ is applied on the entire coupling interface of the fluid analysis. On the structural domain, a constant heat

Table 1 Grid parameters $\left(N_{p}-\right.$ number of points and $N_{\mathrm{cc}}$ - number of cells on the coupling interface)

\begin{tabular}{lcccrrr}
\hline \multicolumn{3}{c}{ Grid } & \multicolumn{3}{c}{ Hot gas } & \multicolumn{3}{c}{ Structure } \\
\hline & \multicolumn{1}{c}{$N_{p}$} & \multicolumn{1}{c}{$N_{\mathrm{cc}}$} & $q_{t}, \mathrm{MW} / \mathrm{m}^{2}$ & \multicolumn{1}{c}{$N_{p}$} & \multicolumn{1}{c}{$N_{\mathrm{cc}}$} & $T_{t}, \mathrm{~K}$ \\
\hline coarse (3) & 114,021 & 2,618 & 57.946 & 36,378 & 3,140 & 1645.914 \\
medium (2) & 259,453 & 5,434 & 59.992 & 250,898 & 13,480 & 1648.294 \\
fine (1) & 590,855 & 11,066 & 60.397 & $1,724,447$ & 53,920 & 1649.105 \\
\hline
\end{tabular}


flux of $q=53 \mathrm{MW} / \mathrm{m}^{2}$ is applied on the coupling surface. The corresponding field variables surface heat flux and surface temperature are extracted from the nodal position at the middle of the cooling channel cross section. These variables are shown in Table 1. The mesh grid size $h$ for this position is evaluated by taking the nodal adjacent cell volumes $\Delta V_{i}$ into account:

$$
h=\left[\frac{1}{N} \sum_{i=1}^{N}\left(\Delta V_{i}\right)\right]^{1 / 3}
$$

Table 2 Data to evaluate a mesh independent solution

\begin{tabular}{lcc}
\hline Parameter & Hot gas & Structure \\
\hline$r_{21}$ & 1.594 & 1.587 \\
$r_{32}$ & 1.624 & 1.710 \\
$p$ & 3.30 & 1.84 \\
$\mathrm{GCI}_{21, \text { fine }}$ & $0.22 \%$ & $0.05 \%$ \\
$\mathrm{GCI}_{32, \text { medium }}$ & $1.08 \%$ & $0.11 \%$ \\
\hline
\end{tabular}

where $N$ is the number of adjacent cells around the point of interest. The grid refinement factor $r=h_{\text {coarse }} / h_{\text {fine }}$ is above 1.5 for all mesh refinements, which is shown in Table 2. For the present FSI analyses, the fine grid of the hot gas side with a GCI of $0.22 \%$ and the medium grid of the structure with a GCI of $0.11 \%$ is chosen. This grid combination is sufficient for a mesh independent solution of the present study.

\subsection{Fluid-Structure Interaction Results}

Two different coating systems are analyzed by the above mentioned FSI analysis approach. The first applied TBC system can be referred to as a conventional setup where a 30-micron bond coat and a 10-micron top coat are defined on top of the hot gas wall. Figure 6 shows the converged temperature distribution of the structural domain. The high temperatures at the combustion chamber inlet can be explained by the assumption of finalized combustion at the inlet, where a homogenous inflow condition neglecting the typical injector head is applied.

Realistic physical conditions are met at the throat region, where the peak temperature reaches about $1516 \mathrm{~K}$. The peak temperature is about $667 \mathrm{~K}$ higher compared to a conventional setup where the copper substrate faces the hot gas directly.

Another small temperature peak can be identified in the nozzle extension just downstream of the manifold position. At this point, a material and geometric cooling channel variation between the coated combustion chamber wall and the nozzle extension is located. The constant heat transfer coefficient in the upstream cooling curcuit are defined five times higher than in the downstream cooling cooling curcuit.

The cut view at the throat region shown on the right in Fig. 6 gives insights about the temperature distribution of the cooling channel cross section. The 


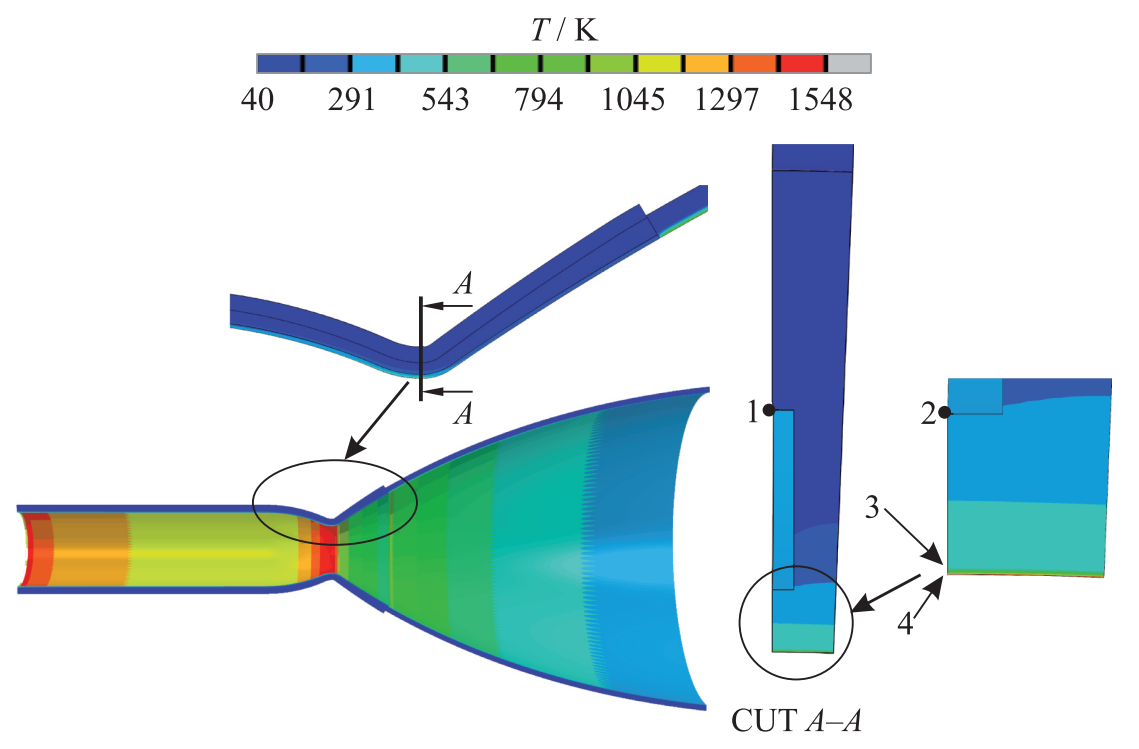

Figure 6 Temperature distribution for the $\mathrm{BC}=30 \mu \mathrm{m}$ and $\mathrm{TBC}=10 \mu \mathrm{m}$ thermal barrier coating at steady state.

Table 3 Temperature distribution (K) at four points defined in Fig. 6 for two different TBC systems at steady state during a hot gas run

\begin{tabular}{lcccc}
\hline \multirow{2}{*}{ Conditions } & \multicolumn{4}{c}{ Position } \\
\cline { 2 - 5 } & 1 & 2 & 3 & 4 \\
\hline $\mathrm{BC}=30 \mu \mathrm{m} ; \mathrm{TBC}=10 \mu \mathrm{m}$ & 50 & 324 & 497 & 1516 \\
$\mathrm{BC}=100 \mu \mathrm{m}$ & 48 & 298 & 456 & 1648 \\
\hline
\end{tabular}

TBC system insulation results in a considerable temperature drop for the copper wall compared to conventional combustion chamber setups. Cross section temperature data for four different positions are defined in Fig. 6 and summarized in Table 3. The maximum copper temperature is reached at position 3 with $476 \mathrm{~K}$ for the first analyzed TBC system.

For the second TBC system analyzed in this study, the top coat layer is omitted, where the bond coat layer is thickened up to $100 \mu \mathrm{m}$. The insulation effect is compareable to the first analyzed TBC system, which can be identified in Table 3. Thicker one-layer TBC systems allow cheaper manufacturing techniques like atmospheric plasma spraying, whereas the individual layers of the first analyzed TBC system are too thin to be manufactured by plasma spraying. 


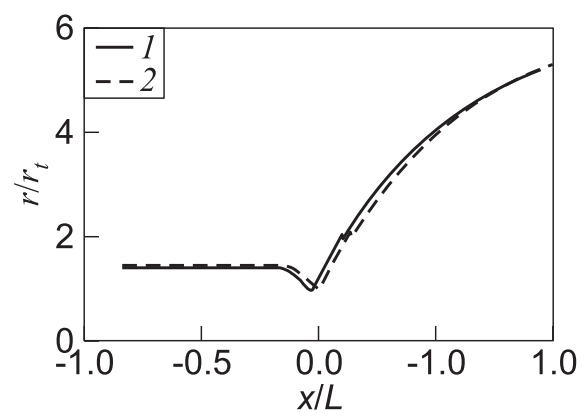

Figure 7 Deformed (1) vs. undeformed (2) thrust chamber contour for the BC = $30 \mu \mathrm{m}$ and $\mathrm{TBC}=10 \mu \mathrm{m}$ thermal barrier coating at steady state

In addition to the thermal results, the global structural response of the steady-state FSI analysis of the first TBC system is shown in Fig. 7. The effect of the global deformed state on the aerothermomechanical analysis is small compared to the strong influence of the thermal interactions.

\section{LOCAL MACROSCALE DELAMINATION MODELING}

Aside from erosion problems investigated by Quentmeyer in [30], it was shown by Miller and Lowell in [31] that TBCs are prone to delamination at the interfaces and even ultimate failure by spallation phenomenon.

The failure phenomenon investigated here includes the effect of macroscopic delamination of TBC systems in rocket thrust chambers. Macroscale delamination modeling is well established in the field of fibrous composites; however, in the field of TBC systems applied to rocket thrust chambers, macroscale delamination growth was not studied so far. This work focuses on the analyses of an FM approach applied to TBC systems. For FM approaches, an initial delamination must be present at which the crack tips are used to determine the fracture mechanical data. An assumed initial macroscale delamination may have an impact on the propagation behavior which must be studied in future.

Delamination propagation analyzed by an FM approach follows directly from the energy release rate $G$ computed by means of linear elastic fracture mechanics for a given loading compared to the critical value $G_{c}$. The fracture energy $G_{c}$ is a material property and, consequently, independent of the loading. Several methods were proposed and validated to compute $G$ or its mode components $G_{I}$, $G_{I I}$, and $G_{I I I}$, e.g., J-Integral, Virtual Crack Extension, Virtual Crack Closure, and Williams [32-34]. 
To gain first insights in the local loading behavior of macroscale delaminations, the Virtual Crack Closure Technique (VCCT) is applied to study sensitivities for two different TBC systems mentioned above.

\subsection{Virtual Crack Closure Technique}

The VCCT is an FM FEM postprocessing approach to compute mixed-mode energy release rates for each fracture mode component. The benefit of the VCCT is to be able to compute the mixed-mode energy release rates by using only one finite element analysis step. Other methods like the crack closure method need two analysis steps, where the crack is physically extended or closed. In the following, the theory and application of the VCCT to 3D linear solid elements is summarized following Krüger [34].

The VCCT is based on two assumptions. The first assumption equals the crack closure method, where it is assumed that the energy needed to extend a crack from length $a$ to the length $a+\Delta a$ is equal to the energy to close the crack by the same length $\Delta a$. The second assumption states that the crack tip configuration shown in Fig. $8 a$ will not alter considerably if a crack is extended to the length $a+\Delta a$ or to $a+2 \Delta a$, meaning that the relative displacements between node $L l$ and node $L l^{*}$ behind the crack with length $a+\Delta a$ will be approximately the same displacement at node $i$, if node $i$ is released and the crack is extended to the length $a+2 \Delta a$.

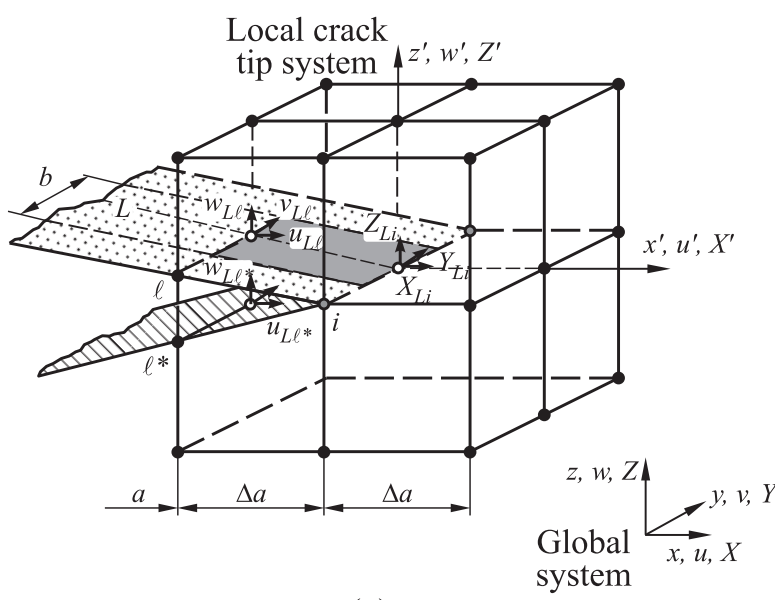

(a)

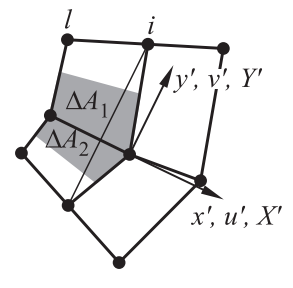

(b)

Figure 8 Local crack tip configuration of an eight node solid element: (a) 3D view [34], and $(b)$ top view 
The energy release rates for the fracture mode components of the eight node solid element shown in Fig. 8 can be evaluated at the crack tip as follows:

$$
\begin{aligned}
G_{I} & =-\frac{Z_{L i}^{\prime}\left(w_{L l}^{\prime}-w_{L l^{*}}^{\prime}\right)}{2\left(\Delta A_{1}+\Delta A_{2}\right)} ; \\
G_{I I} & =-\frac{X_{L i}^{\prime}\left(u_{L l}^{\prime}-u_{L l^{*}}^{\prime}\right)}{2\left(\Delta A_{1}+\Delta A_{2}\right)} \\
G_{I I I} & =-\frac{Y_{L i}^{\prime}\left(v_{L l}^{\prime}-v_{L l^{*}}^{\prime}\right)}{2\left(\Delta A_{1}+\Delta A_{2}\right)}
\end{aligned}
$$

where $X^{\prime}, Y^{\prime}$, and $Z^{\prime}$ are the nodal forces transformed in the local coordinate system $\left(x^{\prime}, y^{\prime}, z^{\prime}\right)$ of the crack tip and $u^{\prime}, v^{\prime}$, and $w^{\prime}$ are the transformed displacements, respectively. Figure $8 b$ shows the area definitions $\Delta A_{1}$ and $\Delta A_{2}$ as well as the definition of the local coordinate system for arbitrary shaped delamination contours.

\subsection{The Parametrized Three-Dimensional Model}

The parametrized 3D delamination model presented in this work consists of the TBC system setup mentioned above. The basic setup of the implemented model consisting of a substrate and the thin layers of bond coat (BC) and top coat (TBC) is shown in Fig. 9a. The parametrized approach is modeled via the preprocessor ABAQUS/CAE. The initial delamination is idealized by the ellipse equation. The special case of a circle can also be chosen as can be seen in Fig. $9 b$. For this work, 8 node linear solid elements are chosen for the complete model.

In case of ideal structures, the local buckle phanomenon of the sublaminates can cause numerical solution difficulties. In order to countervail this effect, a small imperfection can be introduced in the area of delamination, which may also be seen as a realistic effect caused by oxides. The out-of-plane global coordinates inside the delamination area are superimposed with a half cosine wave parallel to the local $\xi, \eta$ coordinate system:

$$
\Delta x_{3}=h_{i} \cos \left(\frac{\pi}{2 a} \xi\right) \cos \left(\frac{\pi}{2 \bar{b}} \eta\right) ; \quad \bar{b}=b \sqrt{1-\frac{\xi^{2}}{a^{2}}} \text { within }\left(\frac{\xi}{a}\right)^{2}+\left(\frac{\eta}{b}\right)^{2}<1
$$

where $h_{i}$ is the maximal imperfection, and $a$ and $b$ are the major and minor semiaxes. This expression assures a tangential plane at the crack front between the intact TBC system and the delamination itself.

Futhermore, the parametrized model features variation in delamination position and orientation according to the global specimen. Multiple delaminations with individual interface positions substrate - BC or BC-TBC are possible as displayed in Fig. 9b. Elliptic representation in parametric coordinates allows for automatic spatial discretization enhancement in areas of large curvature. 


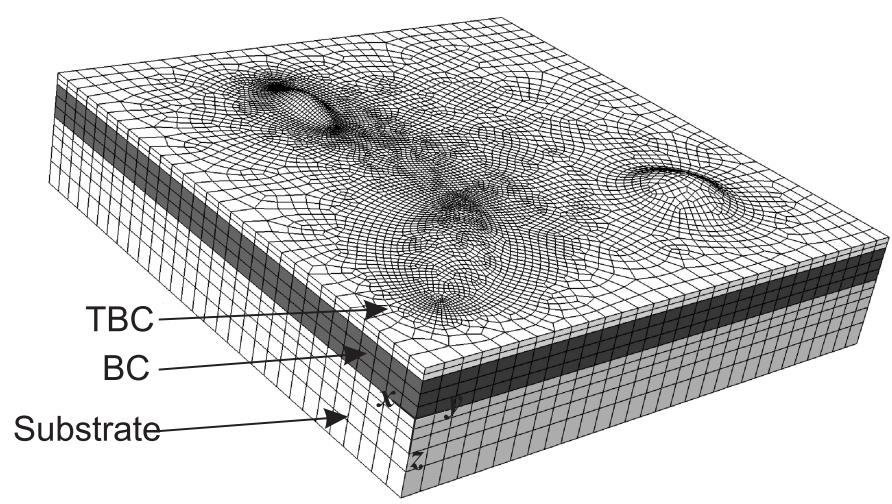

(a)

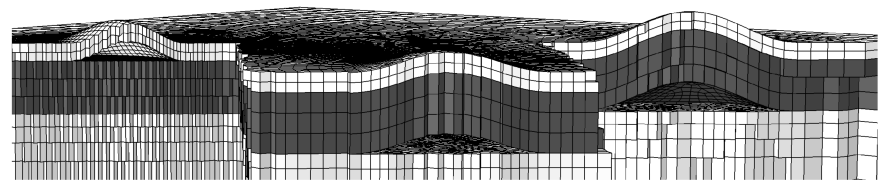

(b)

Figure 9 Three-dimensional delamination model

\subsection{Numerical Results}

In order to get first insights in the local loading conditions of TBC systems, sensitivities of initial delaminations are analyzed. For the finite element analyses of the local approach, displacement boundary conditions are extracted from the global model and applied to the local model as can be seen in Fig. 10.

The width of the local model is defined by one cooling channel at the nozzle throat region of the combustion chamber. The temperature boundary conditions of the copper substrate side are taken from position 2 of Fig. 6 and from position 4 at the hot wall for the top coat side, respectively. For simplicity, to gain first insights, constant temperature and displacement boundary conditions are extracted and applied to the local model. The contraction displacement component $U_{z, G}$ of the chamber wall is extracted at the nozzle throat and applied symmetric for a full cooling channel as $U_{x, L}$ on the local model, which is shown in Fig. 10. In addition, axial contraction of the thrust chamber is extracted for a small segment and applied likewise (see length and width in Table 4).

The failure interface to be analyzed in this study lies between the copper substrate and the bond coat material. This position was identified in experiments to be prone to failure mechanisms [35]. In this study, size variations of delamination circles are analyzed for the above mentioned TBC systems. The 


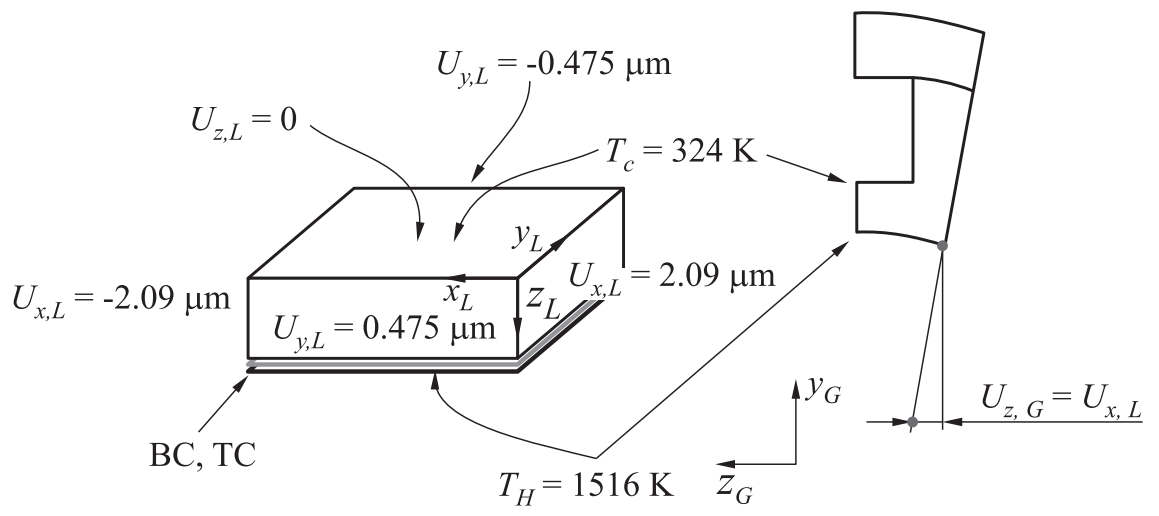

Figure 10 Transfer of boundary conditions from global to local model (BC $=30 \mu \mathrm{m}$; $\mathrm{TBC}=10 \mu \mathrm{m})$

Table 4 Dimensions and boundary conditions applied to the local finite element model

\begin{tabular}{ccccccc}
\hline Conditions & $\begin{array}{c}\text { Length, } \\
\mathrm{m}\end{array}$ & $\begin{array}{c}\text { Width, } \\
\mathrm{m}\end{array}$ & $\begin{array}{c}T_{\mathrm{TBC}}, \\
\mathrm{K}\end{array}$ & $\begin{array}{c}T_{\mathrm{SUB}}, \\
\mathrm{K}\end{array}$ & $\begin{array}{c}U_{x, L}, \\
\mathrm{~m}\end{array}$ & $\begin{array}{c}U_{y, L}, \\
\mathrm{~m}\end{array}$ \\
\hline $\begin{array}{l}\mathrm{BC}=30 \mu \mathrm{m} ; \\
\mathrm{TBC}=10 \mu \mathrm{m}\end{array}$ & 0.00168 & 0.00198 & 1516 & 324 & $\pm 2.09 \cdot 10^{-6}$ & $\pm 4.75 \cdot 10^{-7}$ \\
\hline $\mathrm{BC}=100 \mu \mathrm{m}$ & 0.00096 & 0.00198 & 1648 & 298 & $\pm 2.21 \cdot 10^{-6}$ & $\pm 2.85 \cdot 10^{-7}$ \\
\hline
\end{tabular}

delaminations are positioned in the center of the interface plane of the local model. The copper substrate thickness at the nozzle throat is $1 \mathrm{~mm}$ and the local maximum imperfection hight is defined with $h_{i}=2 \mu \mathrm{m}$.

ABAQUS/Standard is used to solve the sequentially coupled thermomechanical finite element problem. Preanalyses have shown that an element number of 320 along the crack front region is sufficient for the fracture mechanical postprocessing approach. The strainless condition is assumed at $293.15 \mathrm{~K}$, which is the first estimate and needs to be investigated in more detail in future studies.

Results of the fracture mode components $G_{I I}$ and $G_{I I I}$ are shown in Fig. 11. The fracture mode component $G_{I}$ magnitude is about 40 times lower than the $G_{I I}$ and $G_{I I I}$ components; consequently, the loading condition does not result in a considerable opening of the delaminations in mode $I$ direction. Sliding shear in mode $I I$ direction and scissoring shear in mode $I I I$ direction are approximately in equal measure responsible for the failure mechanisms identified in the present sensitivity analyses. As expected, the energy release rates start to elevate by increasing the delamination radius. A 10 percent increased delam- 

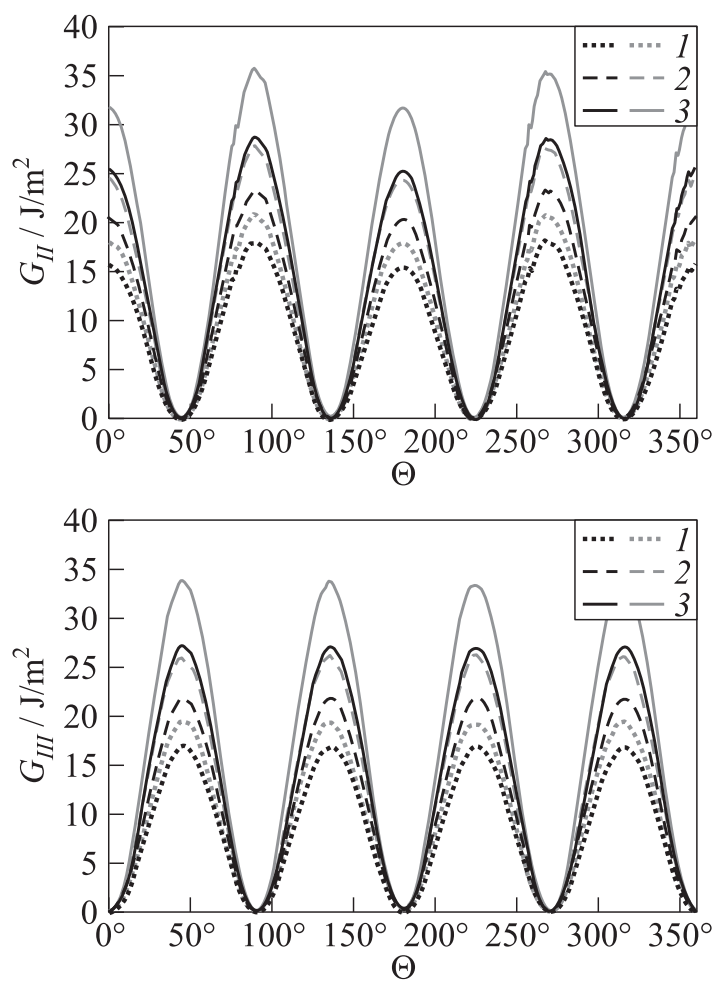

Figure 11 Fracture mode components $G_{I I}$ and $G_{I I I}$ vs. $\Theta$ for two TBC systems (TBC-30-10 - black curves; and BC-100 - grey curevs) and three delamination radii: $1-87.5 \mu \mathrm{m} ; 2-100$; and $3-112.5 \mu \mathrm{m}$

ination radius results roughly in a 25 percent increase of the energy release rate likewise for $G_{I I}$ and $G_{I I I}$.

Comparing the two different TBC systems shown in Fig. 11, one can conclude that the defined one-layer bond coat setup is more critical towards delamination growth compared to the two-layer TBC system setup.

\section{CONCLUDING REMARKS AND OUTLOOK}

A global full parametric 3D modeling approach for cooled rocket thrust chambers has been presented. Thermal and mechanical interactions have been analyzed for the steady-state case of a subscale thrust chamber taking into account two different TBC systems. It was shown by global FSI analyses that one-layer ther- 
mal protection systems are able to achieve the same protection as conventional two-layer TBC systems.

Extracted boundary conditions of the global FSI analyses have been applied to a local FEM to analyze possibe TBC delaminations, which have been investigated by an FM approach. The fracture mode components $I I$ and $I I I$ are identified to have the main influence on the failure mechanisms.

In the ongoing research, the cooling fluid domains analyzed by RANS model will be integrated in the presented fully coupled approach. Furthermore, transient effects will be investigated to address critical loading conditions for applied TBC systems. Thermal transient effects have a great impact on failure mechanisms of TBC systems. Future studies will focus on the application of transient loads and linear variation of boundary conditions applied to the local delamination model.

\section{ACKNOWLEDGMENTS}

Financial support has been provided by the German Science Foundation (DFG Deutsche Forschungsgemeinschaft) in the framework of the Collaborative Research Centre Transregio 40 TP D2 and D3. The authors would like to thank Astrium Space Transportation GmbH, Propulsion \& Equipment for defining the subscale thrust chamber, which was studied in the present work.

\section{REFERENCES}

1. Kuhl, D., A. Woschnak, and O. J. Haidn. 1998. Coupled heat transfer and stress analysis of rocket combustion chambers. AIAA Paper No. 1998-3373.

2. Fischer, S. C., M. Popp, and R. J. Quentmeyer. 1995. Thrust chamber cooling and heat transfer. 2nd Symposium (International) on Liquid Rocket Propulsion.

3. Kuhl, D., J. R. Riccius, and O. J. Haidn. 2000. Thermomechanical fluid-structure analysis and mathermatical optimization techniques applied to cryogenic liquid rocket engine design. Symposium (International) on Liquid Space Propulsion.

4. Liu, Q. Y., E. A. Luke, and P. Cinella. 2005. Coupling heat transfer and fluid flow solvers for multidisciplinary simulations. J. Thermophys. Heat Transfer 19(4):41727 .

5. Knab, O., M. Frey, J. Görgen, C. Maeding, K. Quering, and D. Wiedmann. 2009. Progress in combustion and heat transfer modelling in rocket thrust chamber applied engineering. AIAA Paper No. 2009-5477.

6. Heidmann, J.D., A.J. Kassab, E. A. Divo, A. Eduardo, F. Rodriguez, and E. Steinthorsson. 2003. Conjugate heat transfer effects on a realistic film-cooled turbine vane. ASME Turbo Expo 2003, Power for Land, Sea, and Air Proceedings. 
7. Mangani, L., and C. Bianchini. 2007. Heat transfer application in turbomachinery. 2007 OpenFOAM Conference.

8. Haupt, M. C., R. Niesner, R. Unger, and P. Horst. 2006. Computational aerostructural coupling for hypersonic applications. 9th AIAA/ASME Joint Thermophysics and Heat Transfer Conference. AIAA Paper No. 2006-3252.

9. Haupt, M. C., R. Niesner, R. Unger, and P. Horst. 2008. Numerical analysis of thin-walled structures in hypersonic flow regime. ICAS 2008 Congress.

10. Haupt, M. C., R. Niesner, P. Horst, B. Esser and A. Gülhan. 2009. Computational analysis of a heat transfer experiment including thermally induced deformations. 1st Conference (International) on Computational Methods for Thermal Problems (ThermaComp2009).

11. Park, K., and C. Felippa. 2000. A variational principle for the formulations of partitioned structural systems. Int. J. Numer. Meth. Engng. 47:395-418.

12. Unger, R., M. C. Haupt, and P. Horst. 2007. Application of Lagrange multipliers for coupled problems in fluid and structural interactions. Computers Structures 85:796-809.

13. Shewchuk, J. R. 2002. Delaunay refinement algorithms for triangular mesh generation. Computational Geometry Theory Appl. 22(1-3):21-74.

14. Si, H. 2006. TetGen: A quality tetrahedral mesh generator and a 3D delaunay triangulator. http://tetgen.berlios.de.

15. Rao, G. V. R. 1958. Exhaust nozzle contour for optimum thrust. Jet Propul. 28:37782.

16. Östlund, J. 2002. Flow processes in rocket engine nozzles with focus on flow separation and side-loads. Licentiate Thesis. Royal Institute of Technology, Department of Mechanics, Stockholm.

17. TAU-Code DLR. 2009. Technical Documentation of the DLR TAU-Code. Institut of Aerodynamics and Flow Technology Braunschweig.

18. Ponomarenko, A. 2009. RPA: Design tool for liquid rocket engine analysis. http://www.lpre.de/resources/software/RPA_en.htm.

19. Tahsini, A. M. 2011. Assessment of the accuracy of Spalart-Allmaras turbulence model for application in turbulent wall jets. World Acad. Sci., Engng. Technol. 73:120-25.

20. Wang, Y.Q., P. Jackson, and J. Ackerman. 2006. Numerical investigation of flow over a sphere using LES and the Spalart-Allmaras turbulence model. Comput. Fluid Dynamics J. 15(1):198-205.

21. McAdams, W.H. 1954. Heat transmission. 3rd ed. New York: McGraw-Hill Book Co., Inc. 265-66.

22. Whitaker, S. 1972. Forced convection heat transfer correlations for flow in pipes, past flat plates, single cylinders, single spheres, and flow in packed beds and tube bundles. AlChE J. 18:361-71.

23. Lebedev, V.P., V.V. Lemanov, and V.I. Terekhov. 1999. Heat transfer in a wall jet at high turbulence of cocurrent stream. Int. J. Heat Mass Transfer 42:599-612.

24. Esposito, J. J., and R.F. Zabora. 1997. Thrust chamber life prediction. Vol. I. Mechanical and physical properties of high performance rocket nozzle materials. NASA Report NASA CR-134806. 
25. Freborg, A. M., B. L. Ferguson, W. J. Brindley, and G. J. Petrus. 1998. Modeling oxidation induced stresses in thermal barrier coatings. Mater. Sci. Engng. 245:18290.

26. Deutsches Kupferinstitut. 2005. Kupferdatenblatt CuCr1Zr.

27. Special Metals. 2008. INCONEL alloy 600. http://www.specialmetals.com/products/ inconelalloy600.php.

28. Celik, I. B., U. Ghia, P. J. Roache, C. J. Freitas, H. Coleman, and P. E. Raad. 2008 Procedure for estimation and reporting of uncertainty due to discretization in CFD applications. ASME J. Fluids Eng. 130:078001.

29. Richardson, L., and A. Gaunt. 1927. The deferred approach to the limit. Part I. Single lattice. Part II. Interpenetrating lattices. Philos. Trans. R. Soc. L. A226:299 361.

30. Quentmeyer, R. J. 1977. Experimental fatigue life investigation of cylindrical thrust chambers. AIAA Paper No. 77-893.

31. Miller, R. A., and C.E. Lowell. 1982. Failure mechanisms of thermal barrier coatings exposed to elevated temperatures. Thin Solid Films 95:265-73.

32. Rice, J.R. 1968. A path independent integral and the approximate analysis of strain concentration by notches and cracks. J. App. Mech. 68:379-86.

33. Williams, J. G. 1988. On the calculation of energy release rates for cracked laminates. Int. J. Frac. 36:101-19.

34. Krueger, R. 2002. Virtual crack closure technique: History, approach, and applications. Appl. Mech. Rev. 57:109-43.

35. Schloesser, J., M. Bäker, J. Rösler, and R. Pulz. 2010. Oxidation behavior of thermal barrier coatings on copper substrates. Adv. Sci. Technol. 66:74-79. 